\title{
Resveratrol Modulates Apoptosis and Autophagy Induced by High Glucose and Palmitate in Cardiac Cells
}

\author{
Kui Xua,b Xiu-fen Liu ${ }^{a}$ Zhi-qiang Ke ${ }^{a} \quad$ Qing Yao ${ }^{a}$ Shuang Guo Chao Liu \\ aHubei Key Laboratory of Cardiovascular, Cerebrovascular, and Metabolic Disorders, Hubei University of \\ Science and Technology, Xianning, ${ }^{b}$ Department of Internal Medicine, Hubei University of Science and \\ Technology, Xianning, China
}

\author{
Key Words \\ Resveratrol • Cardiac cell • Autophagy • Apoptosis • AMPK • JNK1
}

\begin{abstract}
Background/Aims: Diabetic cardiomyopathy is associated with increased apoptosis and suppressed autophagy in cardiac cells. The polyphenol resveratrol has shown beneficial effects in various cardiovascular diseases. This study investigated if resveratrol protected cardiac cells by modulating apoptosis and autophagy in the context of diabetes. Methods: H9c2 cardiac myoblast cells were exposed to high glucose combined with palmitate. Autophagy was evaluated by estimating LC3-II/I ratio, P62 protein levels, and LC3 fluorescent puncta. Apoptosis was assessed by using terminal deoxynucleotidyl transferase dUTP nick end labelling (TUNEL), flow cytometry, and analysis of the protein expression of apoptotic markers (cleavage of caspase-3 and PARP). Results: High glucose and palmitate suppressed autophagic activity and exacerbated apoptotic cell death in cardiac myoblast cells. Resveratrol restored autophagy and attenuated apoptosis in cells upon diabetic stimuli. Moreover, resveratrol activated AMPK and JNK1, thereby suppressing mTOR and its downstream effectors p70S6K1 and 4EBP1, as well as disrupting the Beclin1-BCl-2 complex. Conclusion: Resveratrol protects cardiac cells by regulating the switch between autophagy and apoptotic machinery under diabetic conditions, which is attributed by AMPK-mediated phosphorylation of mTORC1/p70S6K1/4EBP1 and JNK-mediated dissociation of Beclin1-Bcl-2. Our study suggests that autophagy may be an important target for resveratrol in the treatment of diabetic cardiomyopathy.
\end{abstract}

(C) 2018 The Author(s)

Published by S. Karger AG, Basel

\section{Introduction}

Diabetes is a leading cause of mortality and morbidity worldwide. More than half of the diabetic patients die from cardiovascular complications [1]. Under diabetic conditions, myocardial cells are exposed to multiple pathological stimuli, such as hyperglycemia,

Kui Xu and Xiu-fen Liu contributed equally to this work. 


\section{Cellular Physiology Cell Physiol Biochem 2018;46:2031-2040 \begin{tabular}{l|l} 
DOI: 10.1159/000489442 & Ond 2018 The Author(s). Published by S. Karger AG, Basel \\
www.karger.com/cpb
\end{tabular}}

Xu et al.: Resveratrol Modulates Cardiac Apoptosis and Autophagy

dyslipidemia, hyperinsulinemia, and oxidative stress [2]. All of which injure cardiac cells, either directly or indirectly, resulting in abnormalities in structure and function, including cardiac apoptosis, interstitial fibrosis, myocardial hypertrophy, and heart failure [3].

Apoptosis is a characteristic feature of diabetic cardiomyopathy [4]. Loss of cardiomyocytes eventually leads to compromised cardiac function due to the very limited proliferative capacity of adult cardiomyocytes. Autophagy is an evolutionarily conserved process for cell survival. It degrades and recycles damaged and long-lived cellular proteins and organelles [5]. Under diabetic conditions, cardiac autophagy is suppressed, suggesting that autophagy may participate in the pathological development of diabetic cardiomyopathy [6]. Both apoptosis and autophagy are critical in maintaining cellular homeostasis. They are discrete cellular processes regulated by distinct groups of regulatory and executioner molecules. However, there are also crosstalk between apoptosis and autophagy [7]. Previous evidence has demonstrated that both processes can be regulated by some common signaling pathways, such as adenosine monophosphate-activated protein kinase (AMPK) and c-Jun $\mathrm{N}$-terminal protein kinase 1 (JNK1). Additionally, the interaction between autophagic protein Beclin1 and the anti-apoptotic protein Bcl-2 represents an important link between autophagy and apoptosis [8].

Resveratrol (Res) is a polyphenol naturally occurring in a variety of plant species such as grapes, cranberries, blueberries, mulberries, and peanuts. As a non-flavonoid polyphenolic compound, it acts as a potent antioxidant and free radical scavenger [9]. A wide range of positive cardiovascular effects of resveratrol have been reported that it stimulates the endothelial production of nitric oxide, inhibits vascular inflammation, prevents platelet aggregation, regulating endoplasmicreticulum stress and reduces oxidative stress [10-12]. Recent advances show that resveratrol regulates autophagy in cardiovascular disorders [13]. We previously reported that resveratrol attenuated high glucose-induced oxidative stress and cardiomyocyte apoptosis through AMPK signaling [14]. In the present study, we sought to investigate the ability of resveratrol to modulate autophagy and apoptosis in cardiac cells under diabetic conditions. The target molecules involved in mediating these effects, such as AMPK and JNK1, were also assessed. Interpreting the interplay between autophagy and apoptosis may also improve our understanding of the pathogenesis of diabetic cardiomyopathy.

\section{Materials and Methods}

\section{Materials}

The H9c2 cardiac myoblast cell line was purchased from Hualianke Biotechnology (Wuhan, China). Fatty-acid-free bovine serum albumin (BSA), palmitate (PA), D-Glucose, 3-Methyladenine (3-MA), Chloroquine (CQ), compound C (CC), SP600125 (SP), and resveratrol (Res) were obtained from Sigma (St. Louis, MO, USA). Green fluorescent protein (GFP)-light chain 3 (LC3) adenovirus was purchased from Hanbio Co. Ltd. (Shanghai, China). Primary antibodies against LC3, P62, AMPK, phospho-AMPK (Thr172), phospho-ACC (Ser79), cleaved caspase3 (C-Casp3), cleaved PARP (C-PARP), Beclin1, Bcl-2, phospho-JNK (Thr183/Tyr185), JNK, phospho-Bcl-2 (Ser70), and $\beta$-actin were purchased from Cell Signaling Technology (Danvers, MA, USA) or Abcam Technology (Cambridge, UK). Dulbecco's modified Eagle's medium (DMEM) was obtained from Hyclone (Logan, Utah, USA). Fetal bovine serum (FBS) was purchased from Gibco (Grand, NY, USA). Other chemicals were purchased from Sigma, unless noted otherwise.

\section{Palmitate solution}

Stock solutions were prepared as follows: palmitic acid (Sigma) was dissolved in $75 \%$ ethanol at $70^{\circ} \mathrm{C}$ at a final concentration of $300 \mathrm{mM}$. Aliquots of stock solutions were blended with fatty-acid-free BSA $(10 \%$ solution in $150 \mathrm{mM} \mathrm{NaCl}$ ) by stirring for $1 \mathrm{~h}$ at $37^{\circ} \mathrm{C}$. The final molar ratio of fatty acid: BSA was 5:1. The final ethanol concentration of stock solution was $1.5 \%(\mathrm{v}: \mathrm{v})$. All control conditions included a solution of vehicle (ethanol: $\mathrm{H}_{2} \mathrm{O}$ ) mixed with fatty-acid-free $\mathrm{BSA}$ in $\mathrm{NaCl}$ solution at the same concentration as the palmitate solution [15]. 


\section{Cellular Physiology Cell Physiol Biochem 2018;46:2031-2040 \begin{tabular}{l|l} 
and Biochemistry Published 10.1159/000489442 & $\begin{array}{l}\text { (c) } 2018 \text { The Author(s). Published by S. Karger AG, Basel } \\
\text { www.karger.com/cpb }\end{array}$ \\
\hline
\end{tabular}}

Xu et al.: Resveratrol Modulates Cardiac Apoptosis and Autophagy

\section{Cell culture and treatments}

H9c2 cells were cultured in DMEM supplemented with $10 \% \mathrm{FBS}, 100 \mathrm{U} / \mathrm{ml}$ of penicillin, and $100 \mathrm{mg} / \mathrm{ml}$ of streptomycin at $37^{\circ} \mathrm{C}$ in a humidified atmosphere $\left(5 \% \mathrm{CO}_{2}\right.$ and $95 \%$ air). Upon reaching $80 \%$ confluence, the cells were incubated in the medium with normal D-glucose $(5.5 \mathrm{mM})$ or high D-glucose concentration (30 mM, HG) combined with palmitate $(0.1 \mathrm{mM}, \mathrm{PA})$ for 24-36 h. Resveratrol (Res, $25 \mu \mathrm{M})$ dissolved in DMSO was added to the medium when the cells were exposed to HG/PA. Pharmacological inhibitors such as 3-methyladenine (3-MA, $10 \mu \mathrm{M}$ ), compound C (CC, $20 \mu \mathrm{M}$ ), or SP600125 (SP, $50 \mu \mathrm{M}$ ) were added to the medium 30 min before the cells were exposed to HG/PA. The final concentration of DMSO in the medium was not more than $0.1 \%(\mathrm{v}: \mathrm{v})$. After experiments, the cells were harvested for analysis.

\section{Immunoprecipitation and western blotting}

After treatment, the cells were washed with ice-cold PBS and harvested in RIPA buffer containing protease/phosphatase inhibitor cocktail (Sigma), $1 \mathrm{mM}$ PMSF (Beyotime, China), and $1 \mathrm{mM} \mathrm{NaVO}{ }_{4}$ at $4^{\circ} \mathrm{C}$. The cell lysates were centrifuged at $12000 \times g$ at $4^{\circ} \mathrm{C}$ for $10 \mathrm{~min}$. The supernatant was collected, and protein concentration was quantified using a BCA Protein Assay Kit (Thermo Fisher). The immunoprecipitates or whole-cell lysates were separated by $8-12 \%$ sodium dodecyl sulfate (SDS)-polyacrylamide gel electrophoresis (SDS-PAGE) and transferred to Immobilon P membranes (Millipore). The membranes were blocked with 5\% skimmed milk (BD) in TBST for $1 \mathrm{~h}$ at room temperature. Primary antibodies were incubated with membranes overnight at $4^{\circ} \mathrm{C}$ and secondary antibodies were incubated for $2 \mathrm{~h}$ at room temperature. After rinsing, the membranes were developed using ECL Plus reagent. Protein bands were measured using the ImageJ software. The background was subtracted from the calculated area.

\section{TUNEL assay}

Cell apoptosis was measured by a TUNEL assay kit (Roche Applied Science, Indianapolis, USA). Briefly, $4 \%$ paraformaldehyde and $0.1 \%$ Triton X-100 were used to fix and permeabilize the cells. After rinsing, the cells were incubated in the TUNEL reagents as described by the manufacturer's instructions. Images were captured under a fluorescence microscope (Olympus BX53, Japan). Six to eight fields were chosen randomly in each section. The apoptotic rate was calculated as the average percentage of TUNEL-positive cells among the total cells.

\section{Flow cytometry assay}

Cell apoptosis was also determined by flow cytometry assay using an Annexin-V FITC/PI staining kit. According to the manufacturer's instructions, the cells were harvested and washed with cold PBS. After centrifugation, the cells were resuspended in binding buffer. The cells were incubated with Annexin-V FITC and propidium iodide (PI) in the dark for $15 \mathrm{~min}$ at room temperature. Stained cells were then analyzed by a FACScan flow cytometer (Becton Dickinson). Data were processed using standard software.

Visualization of autophagic vacuoles

H9c2 cells were transfected with green fluorescent protein (GFP)-light chain 3 (LC3) adenovirus according to the manufacturer's instructions. After $24 \mathrm{~h}$ of transfection, the cells were incubated in normal or $\mathrm{HG} / \mathrm{PA}$ media with or without resveratrol for another $24 \mathrm{~h}$. Chloroquine (CQ, $5 \mu \mathrm{M}$ ) was added to the medium for $16 \mathrm{~h}$, as indicated. Fluorescence images were visualized with a laser scanning confocal microscope (Leica TCS SP8 STED CW, Germany). Autophagy was measured by quantifying the average number of autophagosomes per cell for each sample. A minimum of 100 cells per sample were counted.

\section{Statistical analysis}

Data were collected from repeated experiments and are presented as mean \pm SEM. One-way analysis of variance (ANOVA) and Student-Newman-Keuls Test (SNK) were used for statistical analysis. Differences were considered to be significant at $P$ values $<0.05$.

\section{Results}

Resveratrol prevents autophagy suppression by HG/PA in H9c2 cells

Previous studies demonstrated that basal autophagy is essential for cell survival, and that autophagy is suppressed in diabetic cardiomyopathy. It made us to investigate if 


\section{Cellular Physiology Cell Physiol Biochem 2018;46:2031-2040 \begin{tabular}{c|c|c|} 
DOI: 10.1159/000489442 & O 2018 The Author(s). Published by S. Karger AG, Basel \\
www.karger.com/cpb
\end{tabular} \\ Xu et al.: Resveratrol Modulates Cardiac Apoptosis and Autophagy}

resveratrol protected cardiomyocytes by enhancing autophagy under diabetic conditions. For this purpose, H9c2 cells were exposed to high D-glucose (HG, $30 \mathrm{mM}$ ) combined with palmitate (PA, $0.1 \mathrm{mM}$ ) and treated with resveratrol for 24-36 h. LC3-I, the cytosolic form of the microtubule-associated protein 1A/1B-light chain 3 (LC3), converts to LC3-II by conjugating with phosphatidylethanolamine and translocates to autophagosomes. Thus, the accumulation of LC3-II is regarded as a marker of autophagy [16]. We measured LC3II protein levels using western blot and immunofluorescence, respectively. A decrease in the LC3-II protein level was manifested in H9c2 cells after 24-36 h exposure to HG/PA. In contrast, resveratrol treatment maintained the LC3-II protein level in the H9c2 cells exposed to HG/PA. Similar results were obtained by analysis of GFP-LC3 fluorescence puncta in H9c2 cells. Meanwhile, as the expression of P62 protein is related with autophagic flux [17], we also measured its level in $\mathrm{H} 9 \mathrm{c} 2$ cells which showed that $\mathrm{P} 62$ protein markedly accumulated in the cells exposed to $\mathrm{HG} / \mathrm{PA}$, but it was diminished in the presence of resveratrol. In order to validate the enhanced effect of resveratrol on the autophagic flux, chloroquine (CQ), a lysosome and autophagosome fusion inhibitor [18], was applied on the H9c2 cells. Notably, CQ treatment elevated the protein levels of LC3-II and P62, as well as GFP-LC3 fluorescence puncta in H9c2 cells. Collectively, these data suggest that elevated glucose and palmitate levels reduce autophagic flux, while resveratrol restores the autophagic flux in the presence of HG/PA (Fig. 1).

Resveratrol alleviates apoptosis induced by $H G / P A$ in $H 9 c 2$ cells

Next, we examined apoptotic cell death in H9c2 cells exposed to high D-glucose (HG, $30 \mathrm{mM}$ ) combined with palmitate (PA, $0.1 \mathrm{mM}$ ) levels. As shown in Fig. 2A and $\mathrm{B}$, resveratrol (Res, $25 \mu \mathrm{M}$ ) had little influence on H9c2 cells under normal condition. However, resveratrol treatment markedly alleviated TUNEL-positive cells in the presence of HG/PA. Similar results were obtained in the flow cytometry assay using FITC-annexin V/PI double staining (Fig. 2C and D).

Resveratrol attenuates $H G / P A-$ induced apoptosis associated with enhanced autophagy

In order to study the interaction between autophagy and apoptosis, 3-methylademine (3-MA), an autophagy inhibitor was applied to the H9c2 cells which manipulated autophagic activity by blocking the conversion of LC3-I to LC3-II and autophagosome formation. As expected, resveratrol ameliorated HG/PAinduced alterations in LC3-II formation and P62 accumulation, which were diminished by the addition of 3-MA (Fig. 3A). Meanwhile, resveratrol attenuated the expression of apoptotic markers (cleavage of caspase- 3 and PARP) in the H9c2 cells exposed to HG/PA, which were also abrogated by 3-MA treatment (Fig. 3B). These results indicated that inhibiting autophagy accelerates HG/PA-induced

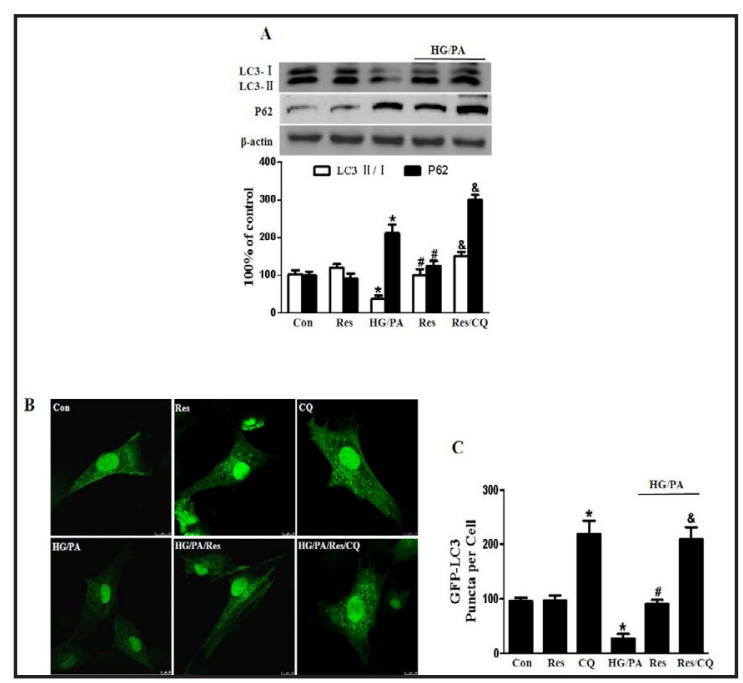

Fig. 1. Resveratrol prevents autophagy inhibited by $\mathrm{HG} / \mathrm{PA}$ in $\mathrm{H} 9 \mathrm{c} 2$ cells. Cells were exposed to high glucose $(\mathrm{HG}, 30 \mathrm{mM})$ combined with palmitate $(\mathrm{PA}$, $0.1 \mathrm{mM}$ ) and treated with resveratrol (Res, $25 \mu \mathrm{M}$ ) for 24-36 h. (A) The protein expression of LC3 II/I and P62 in cell lysates were detected by western blotting. (B) Representative microphotography of GFPLC3 fluorescence puncta. Cells were infected with an adenovirus encoding GFP-LC3 for $24 \mathrm{~h}$. CQ $(5 \mu \mathrm{M})$ was applied on cells for $16 \mathrm{~h}$. GFP-LC3 was detected using a confocal fluorescence microscope. (C) Autophagy was quantified by counting the GFP-LC3 puncta in the cells. $\mathrm{n}=6$; ${ }^{*} \mathrm{P}<0.05$ vs control, ${ }^{\mathrm{P}} \mathrm{P}<0.05$ vs $\mathrm{HG} / \mathrm{PA}$, ${ }^{\circledR} \mathrm{P}<0.05$ vs $\mathrm{HG} / \mathrm{PA} / \mathrm{Res}$. 
apoptotic cell death, and the ability of resveratrol to attenuate apoptosis is closely correlated with the promotion of autophagy.

\section{Resveratrol enhances} the interaction between AMPK and JNK1 in $\mathrm{H} 9 \mathrm{C} 2$ cells exposed to $H G / P A$

Previous studies have suggested that resveratrol activates JNK1 and AMPK in various cells. Consistently, we showed that resveratrol could increase the phosphorylation of AMPK and JNK1 in H9c2 cells in a dose-dependent manner (Fig. 4A). Moreover, coi m munoprecipitation analysis showed that resveratrol significantly improved the direct binding of AMPK and JNK1 in response to HG/ PA stimulation (Fig. 4B), suggesting both kinases might work together to modulate autophagy and apoptosis in resveratroltreated $\mathrm{H} 9 \mathrm{c} 2$ cells.

Resveratrol promotes autophagy by activating the $\quad A M P K / m T O R$ pathway

Based on the critical role of mTOR in autophagy, it was possible that resveratrol activated AMPK and consequently inhibited the mTOR pathway, thus upregulating autophagy [19]. We first validated that AMPK was activated by resveratrol in the H9c2 cells exposed to HG/PA, as monitored by the phosphorylation of AMPK-Thr-172 and ACC-Ser-79, a well-characterized downstream enzyme of AMPK. Additionally, compound C (CC), a potent AMPK inhibitor, not only blocked AMPK activation, but also prevented resveratrol from maintaining LC3-II protein level (Fig. 5A). As a consequence, mTOR signaling was suppressed, as measured by decreased phosphorylation of mTOR (Ser2448) and its downstream effectors, including p70 ribosomal protein S6 kinase 1 (p70S6K1) and 4E-binding protein 1 (4EBP1) (Fig. 5B). Additionally, most of these

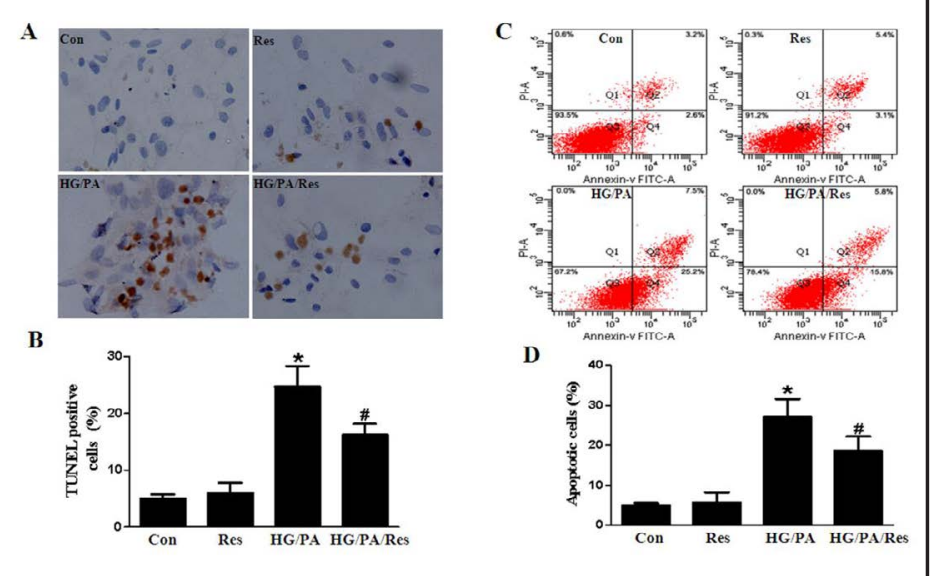

Fig. 2. Resveratrol inhibits apoptosis induced by $\mathrm{HG} / \mathrm{PA}$ in $\mathrm{H} 9 \mathrm{c} 2$ cells. Cells were exposed to high glucose (HG, $30 \mathrm{mM}$ ) combined with palmitate (PA, $0.1 \mathrm{mM}$ ) and treated with resveratrol (Res, $25 \mu \mathrm{M}$ ) for 24-36 h. (A) TUNEL staining was performed; (B) The numbers of TUNEL-positive cells were quantified. (C) and (D) The apoptotic ratios of H9c2 cells in different groups were detected by flow cytometry using FITC-annexin V/PI double staining. (C) Representative diagram of annexin V/PI staining. (D) The apoptotic cells were calculated as the ratio of annexin $\mathrm{V}+/ \mathrm{PI}$ - cells to total cells. $\mathrm{n}=6$; ${ }^{*} \mathrm{P}<0.05$ vs control, ${ }^{\#} \mathrm{P}<0.05$ vs $\mathrm{HG} / \mathrm{PA}$.

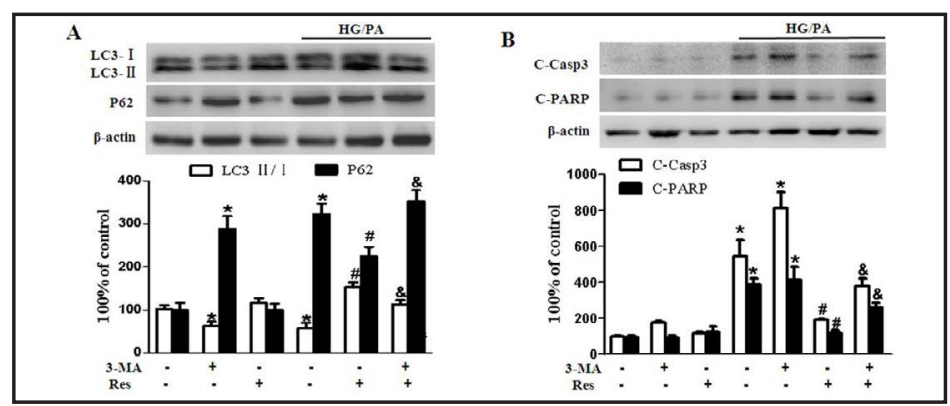

Fig. 3. Resveratrol attenuates HG/PA-induced apoptosis associated with enhanced autophagy. $\mathrm{H} 9 \mathrm{c} 2$ cells were pretreated with 3-methyladenine (3-MA, $10 \mu \mathrm{M}$ ) for $30 \mathrm{~min}$ before exposure to high glucose (HG, $30 \mathrm{mM}$ ) combined with palmitate (PA, $0.1 \mathrm{mM}$ ), and resveratrol (Res, $25 \mu \mathrm{M}$ ) for 24-36 h. (A) The protein expression of LC3 II/I and P62 in cell lysates were detected by western blotting. (B) Apoptosis markers including cleaved caspase-3 (C-Casp3) and cleaved PARP (C-PARP) in cell lysates were detected by western blotting. $\mathrm{n}=4$; ${ }^{*} \mathrm{P}<0.05$ vs control, ${ }^{\#} \mathrm{P}<0.05$ vs HG/PA, ${ }^{\circledR} \mathrm{P}<0.05$ vs HG/PA/Res. 
Fig. 4. Resveratrol activates AMPK and JNK and enhances their association in response to $\mathrm{HG} / \mathrm{PA}$ stimulation. H9c2 cells were exposed to high glucose (HG, $30 \mathrm{mM}$ ) combined with palmitate $(\mathrm{PA}, 0.1 \mathrm{mM})$ and treated with resveratrol (Res, $25 \mu \mathrm{M}$ ) for 24-36 h. (A) AMPK and JNK1 were activated by resveratrol with indicated concentrations. Cells lysates were subjected to western blotting of P-AMPK (Thr172)

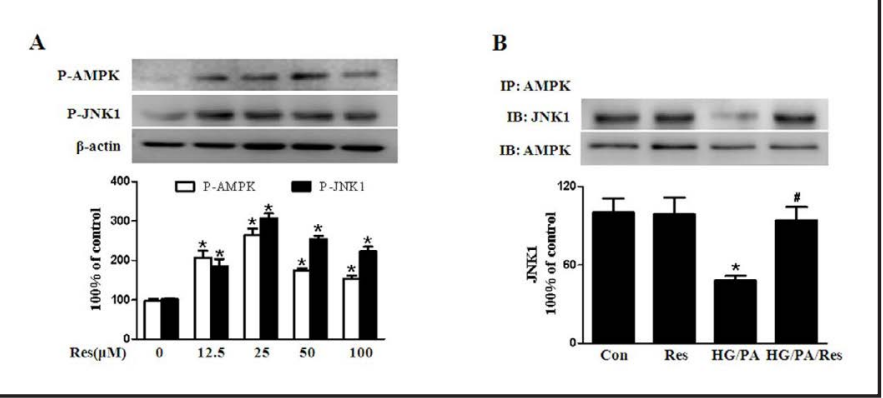
and P-JNK1 (Thr183/Tyr185). (B) Resveratrol enhanced AMPK and JNK1 association in H9c2 cells exposed to HG/PA. The interaction of AMPK and JNK1 was measured by immunoprecipitation (IP) and western blotting (IB). $n=4-5 ;{ }^{*} \mathrm{P}<0.05$ vs control, ${ }^{~} \mathrm{P}<0.05$ vs $\mathrm{HG} / \mathrm{PA}$.

Fig. 5. Resveratrol promotes autophagy through activation of AMPK and inhibition of mTOR pathway. H9c2 cells were pretreated with compound C (CC, $20 \mu \mathrm{M}$ ) for $30 \mathrm{~min}$ before exposure to high glucose ( $\mathrm{HG}, 30 \mathrm{mM}$ ) combined with palmitate (PA, 0.1 $\mathrm{mM})$, and treated with resveratrol (Res, $25 \mu \mathrm{M}$ ) for 24-36 h. (A) Resveratrol activated AMPK and enhanced autophagy in $\mathrm{H} 9 \mathrm{c} 2$ cells exposed to HG/PA. Cell lysates were subjected to measure phosphorylation of AMPK (Thr172) and ACC (Ser79), and pro-

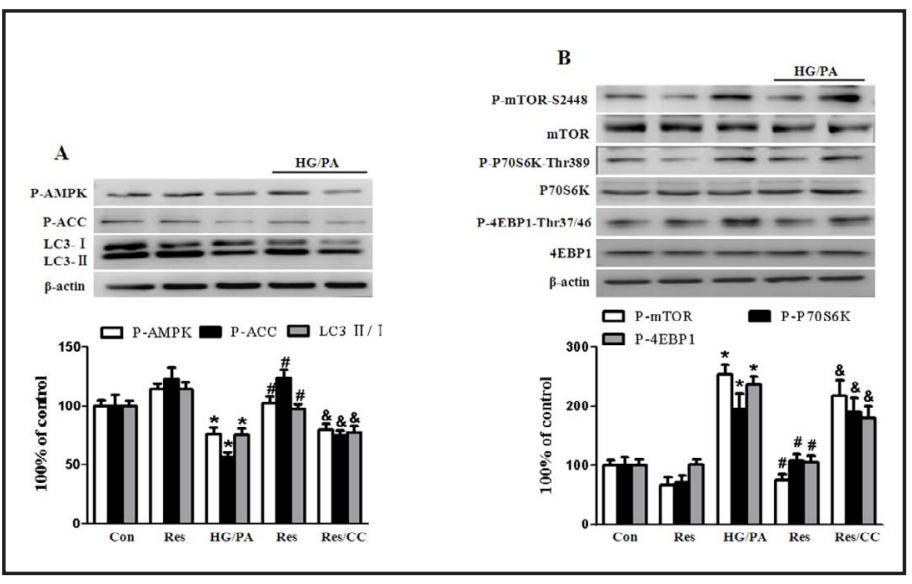
tein levels of LC3 II/I by western blot-

ting. (B) Resveratrol inhibited mTOR pathway in H9c2 cells exposed to HG/PA. mTOR, phosphorylation of mTOR (Ser2448), P70S6K, phosphorylation of P70S6K (Thr389), 4EBP1, and phosphorylation of 4EBP1 (Thr37/46) in cell lysates were detected by western blotting. $\mathrm{n}=4 ;{ }^{*} \mathrm{P}<0.05$ vs control, ${ }^{*} \mathrm{P}<0.05$ vs $\mathrm{HG} / \mathrm{PA}$, \& $\mathrm{P}<0.05$ vs HG/PA/Res.

actions could be reversed by treatment with CC (Fig. 5B). Together, these results suggested the ability of resveratrol to promote autophagy is mediated by AMPK/mTOR pathway.

Resveratrol regulates JNK1-mediated interruption of the Beclin1-Bcl-2 complex, induction of autophagy, and inhibition of apoptosis

Beclin1, a Bcl-2-homology (BH)-3 domain-only protein [20], plays an important role in both autophagosome formation and autolysosome fusion. Beclin1 is also a binding partner of the anti-apoptotic protein Bcl-2 [21]. The binding of Bcl-2 to Beclin1 prevents Bcl-2 from sequestering pro-apoptotic proteins and Beclin 1 from initiating autophagy. This interaction of Beclin1 with Bcl-2 can be modulated by JNK1, which phosphorylates Bcl-2 [22] to disrupt the association between Beclin1 and Bcl-2 [23]. Therefore, we first analyzed JNK1 phosphorylation in $\mathrm{H} 9 \mathrm{c} 2$ cells exposed to HG/PA. As shown in Fig. 6A, the phosphorylation of JNK1 was reduced by HG/PA, and it was restored by resveratrol treatment. However, the JNK1 inhibitor SP600125 diminished the role of resveratrol on JNK1 phosphorylation. Next, coimmunoprecipitation experiments indicated that the binding of Bcl-2 to Beclin1 was enhanced in the presence of $\mathrm{HG} / \mathrm{PA}$, but this effect was lessened by resveratrol treatment. Additionally, treatment with SP600125 restored the binding of Beclin1 to Bcl-2 (Fig. 6B). In line with JNK1 activation, resveratrol enhanced Bcl-2 phosphorylation at Ser70, and this effect was weakened by SP600125 (Fig. 6C). As a result, autophagy and apoptosis, which were 


\section{Cellular Physiology Cell Physiol Biochem 2018;46:2031-2040 \begin{tabular}{ll|l} 
DOI: 10.1159/000489442 & $\begin{array}{l}\text { O 2018 The Author(s). Published by S. Karger AG, Basel } \\
\text { www.karger.com/cpb }\end{array}$ \\
\hline and Biochemistry
\end{tabular}

Fig. 6. Resveratrol regulates JNK-mediated interruption of the Beclin1-Bcl-2 complex, induction of autophagy, and inhibition of apoptosis. H9c2 cells were pretreated with SP600125 (SP, $50 \mu \mathrm{M}$ ) for 30 min before exposure to high glucose ( $\mathrm{HG}, 30 \mathrm{mM})$ combined with palmitate (PA, $0.1 \mathrm{mM}$ ) and treated with resveratrol (Res, $25 \mu \mathrm{M}$ ) for 24-36 h. (A) Resveratrol activated JNK1 in $\mathrm{H} 9 \mathrm{c} 2$ cells exposed to HG/PA. Phosphorylation of JNK1 (Thr183/Tyr185) and JNK1 were measured in cell lysates by western blotting. (B) Beclin1 or Bcl-2 was immunoprecipitated (IP) from cell lysates, and Bcl-2 or Beclin1 in the immunoprecipitate was detected by western blotting (IB). (C) Cell lysates were immunoprecipitated with anti-Bcl-2 antibody and then subjected to immunoblot using P-Bcl-2 (Ser70) and Bcl-2 antibodies. (D) LC3-II/I protein levels in cells lysates were measured by western blotting. (E) Apoptosis markers including cleaved caspase-3 (C-Casp3) and cleaved PARP (C-PARP) in cell lysates were detected by western blotting. $\mathrm{n}=3-5$; ${ }^{*} \mathrm{P}<0.05$ vs control, " $\mathrm{P}<0.05$ vs HG/PA, ${ }^{\&} \mathrm{P}<0.05$ vs $\mathrm{HG} / \mathrm{PA} /$ Res.

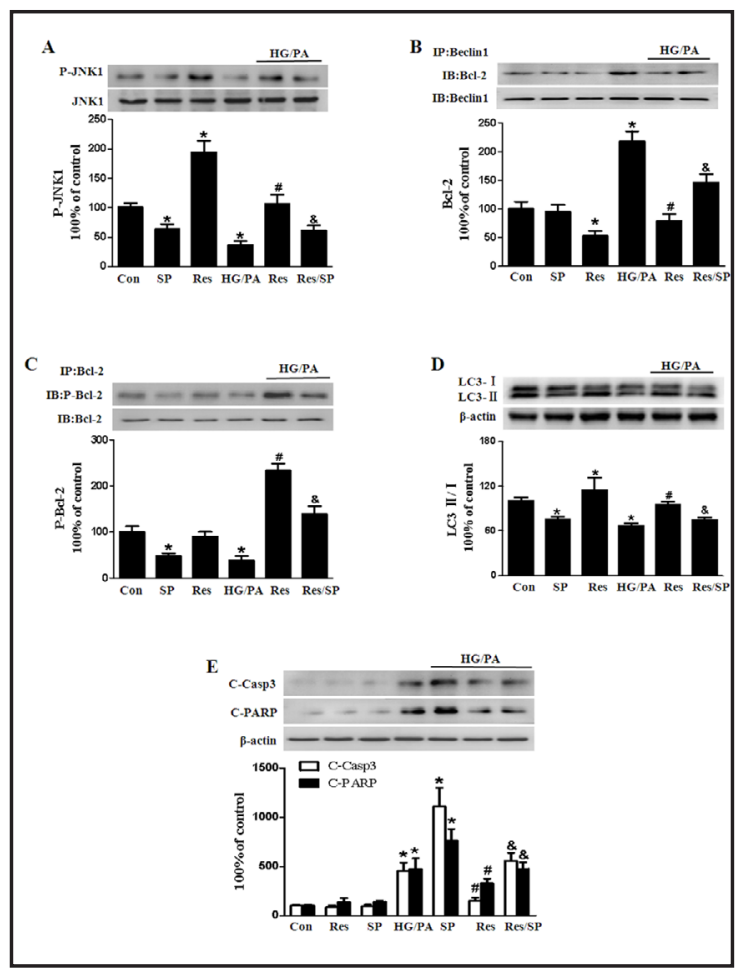

evaluated by the protein levels of LC3-II and apoptotic markers (i.e., cleavage of caspase-3 and PARP), were inversely regulated by resveratrol but reversed by SP600125 in cardiac cells exposed to HG/PA (Fig. 6D and E). These results suggest that JNK1 activation is critical for resveratrol to promote autophagy and prevent apoptosis in cardiac cells in response to diabetic stimuli.

\section{Discussion}

Resveratrol is a polyphenol phytoalexin contained in a variety of plant species. It has been implicated in explanation for the cardiovascular benefits of red wine. Resveratrol protects against ischemic heart disease, hypertension [24], cardiac hypertrophy [25], and atherosclerosis in animal models of cardiovascular disease. The present study provided evidence that resveratrol protects against diabetic cardiomyopathy by activating AMPK and JNK1 pathway to modulate the switch between autophagy and apoptotic machinery. Our results are supported by previous studies that autophagy is suppressed in type 2 diabetes and resveratrol acts as an regulator of autophagy $[8,13]$ and apoptosis [26].

Diabetic cardiomyopathy is an independent cardiovascular disease characterized by ventricular dysfunction in the absence of coronary artery disease, hypertension, or valvular diseases [27]. The metabolic disorders in diabetes, such as hyperglycemia and hyperlipidemia, exert multiple toxin on the myocardium resulting in cell apoptosis and death. Evidence from clinical and animal studies have demonstrated that increased apoptosis is a common feature in diabetic cardiomyopathy $[28,29]$. In contrast to non-diabetic individuals, apoptosis was increased in diabetic patients by 85 folds, 61 folds, and 26 folds in cardiomyocytes, endothelial cells, and fibroblasts, respectively [6]. The adult cardiomyocytes rarely proliferate so that the loss of cardiomyocytes would eventually lead to compromised cardiac function. On the other hand, autophagy also plays important roles in determining cellular fate. As an evolutionarily conserved cellular housekeeping process, autophagy is not only essential for the degradation and disposal of damaged organelles and proteins, but also for recycling cellular substrates for energy generation and cell survival. A basal level of autophagy is required for cellular 


\section{Cellular Physiology Cell Physiol Biochem 2018;46:2031-2040 \\ \begin{tabular}{c|c} 
DOI: 10.1159/000489442 & O 2018 The Author(s). Published by S. Karger AG, Basel \\
www.karger.com/cpb
\end{tabular}}

Xu et al.: Resveratrol Modulates Cardiac Apoptosis and Autophagy

function. And suppressed autophagy below physiological levels leads to heart failure with enhanced protein aggregation, suggesting that autophagy is critical to maintain normal heart function [30]. In consistent with previous reports, our present study showed that autophagy was suppressed in cardiac cells upon diabetic stimuli. Meanwhile, apoptotic cell death was markedly exacerbated in autophagy-deficient cardiac cells. We revealed that resveratrol prevented cardiac cells from HG/PA-induced apoptosis by promoting autophagic activity. To the best of our knowledge, this is the first evidence that resveratrol protects cardiac cells via modulation of autophagy and apoptosis under diabetic conditions.

We have previously reported that resveratrol attenuates high glucose-induced cardiomyocyte apoptosis through AMPK. AMPK is a serine/threonine kinase that senses cellular energy status and regulates energy homeostasis [31]. Activation of AMPK is involved in determining multiple cellular processes, including cell growth, apoptosis [32], and autophagy [33]. It is known that the activation of AMPK could inhibit mTOR, the bestcharacterized protein kinase that negatively regulates autophagy [19]. In the present study, we demonstrated that resveratrol activated AMPK and suppressed mTOR activation in cardiac cells under diabetic conditions, as evidenced by the phosphorylation of mTOR and its downstream components in the mTOR complex [18]. Thus, one likely reason for resveratrol to improve cardiac autophagy is the activation of AMPK.

Beclin1, a mammalian ortholog of yeast Atg6, is a core component of the autophagy machinery. It recruits an activated class III PI3K (Vps34), which plays a central role in initiating autophagy. Bcl-2 is a well-known anti-apoptotic protein. It protects cells against apoptosis by preserving the integrity of the mitochondrial outer membrane and inhibiting the release of cytochrome $\mathrm{C}$ into the cytoplasm. Beclin1 physically interacts with Bcl2. The binding of Bcl-2 to Beclin1 inhibits Beclin1-mediated autophagy by sequestering Beclin1 away from class III PI3K. This interaction between Beclin1 and Bcl-2 represents an important convergent point for the apoptotic and autophagic machineries [34]. Moreover, this interaction can be modulated by phosphorylation of Bcl-2 by JNK1, thereby promoting the dissociation of Beclin1 from Bcl-2, which, in turn, inducing autophagy $[23,35]$. Our present study revealed that resveratrol activated JNK1 and phosphorylated Bcl-2, and thus disrupting the association between Bcl-2 and Beclin1. As a consequence, free Beclin1 binds to class III PI3K to induce autophagy, and free Bcl-2 binds to pro-apoptotic proteins to inhibit apoptosis. Additionally, AMPK and JNK1 are not apart from each other. Our data showed that AMPK directly bound with JNK1 in the cultured H9c2 cardiac cells. It was shown that high levels of glucose and palmitate could interrupt the association between both protein kinases, whereas resveratrol maintained their connection in cardiac cells. Our results are consistent with previous report that AMPK binds to JNK1 and acts as an upstream kinase [36]. Although their relationship needs to be further dissected, the presented data suggest that AMPK and JNK1 work together to modulate autophagy and apoptosis in cardiac cells under diabetic conditions.

Taken together, we have demonstrated that resveratrol protects cardiac cells by regulating the switch between autophagy and apoptotic machinery upon diabetic stimulation, which is attributed by AMPK-mediated phosphorylation of the mTOR/p70S6K1/4EBP1 pathway and JNK1-mediated dissociation of the Beclin1-Bcl-2 complex. Our study has not only shed new insight into the beneficial effects of resveratrol on diabetic cardiomyopathy, but also revealed that modulating autophagy may represent an effective strategy for the treatment of cardiovascular diseases associated with diabetes.

\section{Abbreviations}

AMPK (adenosine monophosphate-activated protein kinase); JNK1 (c-Jun N-terminal protein kinase 1); mTORC(mammalian target of rapamycin); BSA (bovine serum albumin); PA (palmitate), HG (high D-Glucose); 3-MA (3-Methyladenine); CQ (Chloroquine); CC (compound C); SP (SP600125); Res (resveratrol); GFP (Green fluorescent protein). 


\section{Cellular Physiology Cell Physiol Biochem 2018;46:2031-2040 \begin{tabular}{l|l} 
DOI: 10.1159/000489442 & O 2018 The Author(s). Published by S. Karger AG, Basel \\
www.karger.com/cpb
\end{tabular} \\ Xu et al.: Resveratrol Modulates Cardiac Apoptosis and Autophagy}

\section{Acknowledgements}

This work was supported by grants from the Outstanding Youth Scientific Innovation Team Project for the Universities of Hubei Province [T201213], New Century Excellent Talents Project of the Ministry of Education [NCET-13-0781], and Hubei Major Projects of Technical Innovation [2016ACA148].

XK and LXF participated in the design of the study and conducted experiments, analyzed data, performed the statistical analysis, and wrote draft of the manuscript; KZQ and YQ were involved in performing experiments; GS was involved in revising the manuscript; LC conceived of the study, designed and coordinated the study, and wrote manuscript. All authors read and approved the final manuscript.

\section{Disclosure Statement}

No conflict of interests exists.

\section{References}

1 Lee C, Joseph L, Colosimo A, Dasgupta K: Mortality in diabetes compared with previous cardiovascular disease: a gender-specific meta-analysis. Diabetes Metab 2012;38:420-427.

2 Mizamtsidi M, Paschou SA, Grapsa J, Vryonidou A: Diabetic cardiomyopathy: a clinical entity or a cluster of molecular heart changes? Eur J Clin Invest 2016; 46:947-953.

3 Stratmann B, Stork I, Tschoepe D: [Diabetic cardiomyopathy--a type of coronary artery disease?]. Internist (Berl) 2008;49:436-440.

4 Kuethe F, Sigusch HH, Bornstein SR, Hilbig K, Kamvissi V, Figulla HR: Apoptosis in patients with dilated cardiomyopathy and diabetes: a feature of diabetic cardiomyopathy? Horm Metab Res 2007;39:672-676.

5 Lavandero S, Chiong M, Rothermel BA, Hill JA: Autophagy in cardiovascular biology. J Clin Invest 2015;125:55-64.

6 Li ZL, Woollard JR, Ebrahimi B, Crane JA, Jordan KL, Lerman A, Wang SM, Lerman LO: Transition from obesity to metabolic syndrome is associated with altered myocardial autophagy and apoptosis. Arterioscler Thromb Vasc Biol 2012; 32:1132-1141.

7 Nishida K, Yamaguchi O, Otsu K: Crosstalk between autophagy and apoptosis in heart disease. Circ Res 2008;103:343-351.

8 Wei Y, Sinha S, Levine B: Dual role of JNK1-mediated phosphorylation of Bcl-2 in autophagy and apoptosis regulation. Autophagy 2008;4:949-951.

-9 Lorenz P, Roychowdhury S, Engelmann M, Wolf G, Horn TF: Oxyresveratrol and resveratrol are potent antioxidants and free radical scavengers: effect on nitrosative and oxidative stress derived from microglial cells. Nitric Oxide 2003;9:64-76.

10 Bonnefont-Rousselot D: Resveratrol and Cardiovascular Diseases. Nutrients DOI: 10.3390/nu8050250.

-11 Madreiter-Sokolowski CT, Gottschalk B, Parichatikanond W, Eroglu E, Klec C, Waldeck-Weiermair M, Malli R, Graier WF: Resveratrol Specifically Kills Cancer Cells by a Devastating Increase in the Ca2+ Coupling Between the Greatly Tethered Endoplasmic Reticulum and Mitochondria. Cell Physiol Biochem 2016;39:1404-1420.

12 Feng Y, Yang Y, Fan C, Di S, Hu W, Jiang S, Li T, Ma Z, Chao D, Feng X, Xin Z, Pang S, Li X, Yan X: Pterostilbene Inhibits the Growth of Human Esophageal Cancer Cells by Regulating Endoplasmic Reticulum Stress. Cell Physiol Biochem 2016;38:1226-1244.

13 Hashemzaei M, Entezari Heravi R, Rezaee R, Roohbakhsh A, Karimi G: Regulation of autophagy by some natural products as a potential therapeutic strategy for cardiovascular disorders. Eur J Pharmacol 2017;802:44-51.

14 Guo S, Yao Q, Ke Z, Chen H, Wu J, Liu C: Resveratrol attenuates high glucose-induced oxidative stress and cardiomyocyte apoptosis through AMPK. Mol Cell Endocrinol 2015;412:85-94. 


\section{Cellular Physiology Cell Physiol Biochem 2018;46:2031-2040

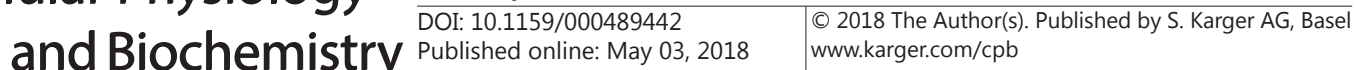

15 Hoshino A, Ariyoshi M, Okawa Y, Kaimoto S, Uchihashi M, Fukai K, Iwai-Kanai E, Ikeda K, Ueyama T, Ogata T, Matoba S: Inhibition of p53 preserves Parkin-mediated mitophagy and pancreatic beta-cell function in diabetes. Proc Natl Acad Sci USA 2014;111:3116-3121.

16 Tanida I, Ueno T, Kominami E: LC3 conjugation system in mammalian autophagy. Int J Biochem Cell Biol 2004;36:2503-2518.

17 Komatsu M, Kageyama S, Ichimura Y: P62/SQSTM1/A170: physiology and pathology. Pharmacol Res 2012;66:457-462.

18 Mizushima N, Yoshimori T, Levine B: Methods in mammalian autophagy research. Cell 2010;140:313-326.

19 Shaw RJ: LKB1 and AMP-activated protein kinase control of mTOR signalling and growth. Acta Physiol(Oxf) 2009;196:65-80.

20 Oberstein A, Jeffrey PD, Shi Y: Crystal structure of the Bcl-XL-Beclin1 peptide complex: Beclin1 is a novel BH3-only protein. J Biol Chem 2007;282: 13123-13132.

-21 Liang XH, Kleeman LK, Jiang HH, Gordon G, Goldman JE, Berry G, Herman B, Levine B: Protection against fatal Sindbis virus encephalitis by beclin, a novel Bcl-2-interacting protein. J Virol 1998;72:8586-8596.

-22 Strappazzon F, Vietri-Rudan M, Campello S, Nazio F, Florenzano F, Fimia GM, Piacentini M, Levine B, Cecconi F: Mitochondrial BCL-2 inhibits AMBRA1-induced autophagy. EMBO J 2011;30:1195-1208.

23 Wei Y, Pattingre S, Sinha S, Bassik M, Levine B: JNK1-mediated phosphorylation of Bcl-2 regulates starvation-induced autophagy. Mol Cell 2008;30:678-688.

24 Yu L, Tu Y, Jia X, Fang K, Liu L, Wan L, Xiang C, Wang Y, Sun X, Liu T, Yu D, Cao W, Song Y, Fan Y: Resveratrol Protects Against Pulmonary Arterial Hypertension in Rats via Activation of Silent Information Regulator 1. Cell Physiol Biochem 2017;42:55-67.

25 Lin Y, Zhu J, Zhang X, Wang J, Xiao W, Li B, Jin L, Lian J, Zhou L, Liu J: Inhibition of Cardiomyocytes Hypertrophy by Resveratrol Is Associated with Amelioration of Endoplasmic Reticulum Stress. Cell Physiol Biochem 2016; 39:780-789.

-26 Tian Z, Wang J, Xu M, Wang Y, Zhang M, Zhou Y: Resveratrol Improves Cognitive Impairment by Regulating Apoptosis and Synaptic Plasticity in Streptozotocin-Induced Diabetic Rats. Cell Physiol Biochem 2016;40: 1670-1677.

-27 Teupe C, Rosak C: Diabetic cardiomyopathy and diastolic heart failure -- difficulties with relaxation. Diabetes Res Clin Pract 2012;97:185-194.

28 Chowdhry MF, Vohra HA, Galinanes M: Diabetes increases apoptosis and necrosis in both ischemic and nonischemic human myocardium: role of caspases and poly-adenosine diphosphate-ribose polymerase. J Thorac Cardiovasc Surg 2007;134:124-131.

29 Li Z, Zhang T, Dai H, Liu G, Wang H, Sun Y, Zhang Y, Ge Z: Involvement of endoplasmic reticulum stress in myocardial apoptosis of streptozocin-induced diabetic rats. J Clin Biochem Nutr 2007;41:58-67.

-30 Nakai A, Yamaguchi O, Takeda T, Higuchi Y, Hikoso S, Taniike M, Omiya S, Mizote I, Matsumura Y, Asahi M, Nishida K, Hori M, Mizushima N, Otsu K: The role of autophagy in cardiomyocytes in the basal state and in response to hemodynamic stress. Nat Med 2007;13:619-624.

31 Hardie DG: AMP-activated/SNF1 protein kinases: conserved guardians of cellular energy. Nat Rev Mol Cell Biol 2007;8:774-785.

-32 Shirwany NA, Zou MH: AMPK in cardiovascular health and disease. Acta Pharmacol Sin 2010;31:10751084.

33 Herzig S, Shaw RJ: AMPK: guardian of metabolism and mitochondrial homeostasis. Nat Rev Mol Cell Biol 2018;19:121-135.

-34 Pattingre S, Tassa A, Qu X, Garuti R, Liang XH, Mizushima N, Packer M, Schneider MD, Levine B: Bcl-2 antiapoptotic proteins inhibit Beclin1-dependent autophagy. Cell 2005;122:927-939.

-35 He C, Bassik MC, Moresi V, Sun K, Wei Y, Zou Z, An Z, Loh J, Fisher J, Sun Q, Korsmeyer S, Packer M, May HI, Hill JA, Virgin HW, Gilpin C, Xiao G, Bassel-Duby R, Scherer PE, Levine B: Exercise-induced BCL2-regulated autophagy is required for muscle glucose homeostasis. Nature 2012;481: 511-515.

-36 He C, Zhu H, Li H, Zou MH, Xie Z: Dissociation of Bcl-2-Beclin1 complex by activated AMPK enhances cardiac autophagy and protects against cardiomyocyte apoptosis in diabetes. Diabetes 2013;62:1270-1281. 